\title{
Self-Identification Algorithm for Zeolite-based thermal capacity Gas Sensor
}

\author{
M. Pouliquen (1) • M. Denoual (1,6) • C. Jorel (1) • C. Radu (1) • D. Robbes (1) • J. Grand (2) \\ - H. Awala (2) . S. Mintova (2) . M. Harnois (3) • O. de Sagazan (3) - S. Inoue (4) E. \\ Lebrasseur $^{(4)}$ • K. Yamada ${ }^{(4)}$. Y. Okamoto ${ }^{(4)}$ • A. Mita-Tixier ${ }^{(5,6)}$ • Y. Mita ${ }^{(4,6)}$
}

\begin{abstract}
We demonstrate a new operation mode of thermal gas sensor based on thermal capacity extraction with identification algorithm. The system is a silicon microstructure covered with zeolites operated at constant temperature while stimulated by heat pseudorandom sequence. The proposed detection principle is demonstrated at room temperature and atmospheric pressure through the detection of gas water molecules with an hydrophilic FAU-type zeolite coating. The identification algorithm is a continuous-time closed-loop identification algorithm based on the instrumental variable principle.
\end{abstract}

\section{(1)}

GREYC-UMR6072

ENSICAEN, University of Caen

Caen, France

E-mail: mathieu.pouliquen@unicaen.fr

(2)

LCS-UMR6506

ENSICAEN, University of Caen

Caen, France

(3)

IETR-microcapteur-UMR6164

University of Rennes

Rennes, France

(4)

iSML

University of Tokyo

Tokyo, Japan

(5)

RCAST

University of Tokyo

Tokyo, Japan

(6)

LIMMS-UMI2820

University of Tokyo

Tokyo, Japan
Keywords system identification algorithm $\cdot$ thermal capacity gas sensor $\cdot$ zeolites

\section{Introduction}

In literature of MEMS gas sensors, on one hand mechanical devices are proposed to monitor mass variation due to gas adsorption ([1]). On the other hand, techniques based on thermal analysis either require the catalytic decomposition and/or desorption of molecules as a function of temperature with thermal cycles ([2], $[3])$; or they rely on gas thermal conductance measurement. In this work, the principle is based on thermal capacity extraction. This principle was demonstrated in previous work in time domain ([4]) and frequency domain with the particular thermal resonant operation mode $([5])$. In this paper, thermal capacity extraction is performed with identification algorithm while the thermal system is operated in closed-loop mode and kept at constant temperature and self-stimulated with pseudorandom heat pulses. Constant temperature operation of the device can be favorable as it reduces convection around the tip of the device.

About identification, we are interested in the estimation of a continuous-time model so as to extract the thermal capacity from the model parameters. The main difficulty in closed-loop identification is due to the correlation between the noise and the control signal, induced by the loop ([6], [7]). Different specific identification methods have been developed for the closed-loop identification. In the context of the identification of a continuous-time model, the method "Closed Loop Simplified Refined Instrumental Variable Continuous-time" (CLSRIVC) identification method presented in ([8]) has 
two advantages. First, it allows the direct unbiased estimation of the parameters of the continuous-time model without the need to estimate a discrete time model. Second, it allows the estimation of the variance of these estimated parameters and consequently the evaluation of the reliability of such estimates.

Experimental data are acquired using a micro-fabricated thermal micro-device associated to zeolite material. Zeolites are used as a selective layers as in other works in literature ([9]). Zeolites are micro/nanoporous materials that reveal great chemical, size and shape selectivity enabling detection and differentiation between different gases and vapors.

\section{Description of the system and set-up}

\subsection{General description of the system}

The system comprises a thermal micro-device, conditioning electronics with gain $G_{e}$, a microcontroller and an identification algorithm (Fig. 1).

The thermal micro-device is a first order thermal system whose time constant, $\tau_{t h}$, depends on gas adsorption. The continuous-time model takes the form,

$G(p)=\frac{G_{0} G_{e}}{1+\tau_{t h} p}$

with $p$ the time-domain differentiation operator.

The microcontroller implements a PI-controller (Proportional-Integral) that turns the system into a second order system that is tuned to regulate the temperature of the thermal micro-device.

$H(p)=\frac{G_{0}}{1+\left(\frac{1+G_{0} G_{e} k_{p}}{G_{0} G_{e} k_{i}}\right) p+\left(\frac{\tau_{t h}}{G_{0} G_{e} k_{i}}\right) p^{2}}$

The natural pulsation of the second order system depends on the thermal micro-device time constant and consequently on the adsorbed gas. Therefore, the determination of the parameters of the second order system can be used to derive the adsorbed gas variations. In order to extract the parameters while operating in closedloop mode, the micro-controller generates a pseudorandom Joule power stimulus.

In order to extract the thermal parameters of the thermal device, the CLSRIVC identification method is run on a computer. The algorithm inputs are the PRBS binary sequence (so as to satisfy the persistent excitation condition) and the voltage representative of the device temperature. The algorithm realizes the estimation of the parameters of the continuous-time model (i.e. $G_{0} G_{e}$ and $\tau_{t h}$ ) of the device and the estimation of the variance on these estimated parameters.

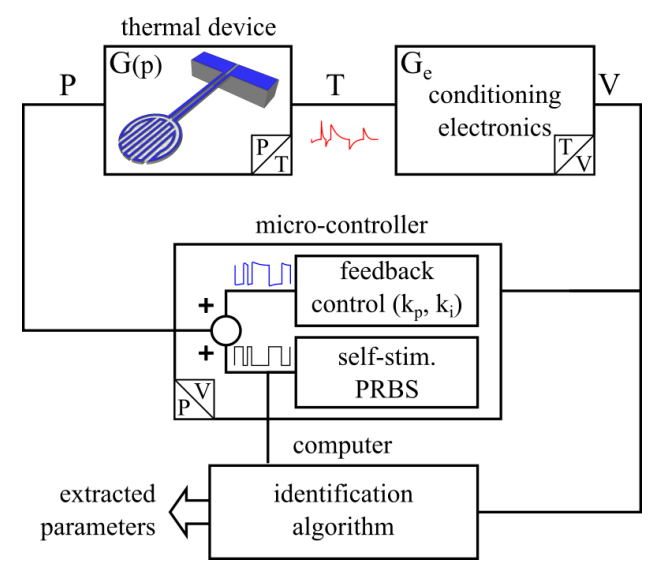

Fig. 1 Block diagram of the operation mode. P: Joule power, $\mathrm{T}$ : temperature of the tip of the device, $\mathrm{V}$ : voltage at the output of the conditioning electronics representative of the temperature. PRBS: Pseudo-Random Binary Sequence. The thermal device is operated in closed-loop mode thanks to a heat feedback controlled by a conventional proportional-integral (PI) controller implemented in a micro-controller. The PI controller is set to keep constant the temperature of the thermal device. The micro-controller also produces a pseudo-random binary sequence that thermally stimulates the thermal device through heat random pulses.

\subsection{Description of the thermal micro-device}

The thermal device consists of a cantilever like structure with a beam and a disk tip filled with zeolites (Fig. 2). The beam with thermal conductivity $\left(G_{t h}\right)$ insulates the thermal capacity of the disk tip $\left(C_{t h}\right)$ from the surrounding. The tip and the beam form a first order thermal system with time constant $\tau_{t h}=\frac{C_{t h}}{G_{t h}}$. A single aluminum resistor on the device is used both to monitor the temperature of the tip and to produce heat. Heat is produced by a feedback path to operate the system in closed-loop mode and for heat stimulation.
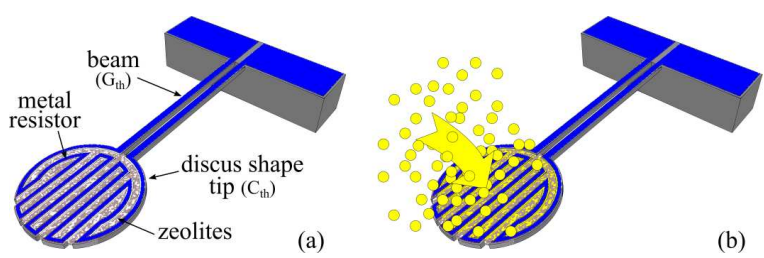

Fig. 2 (a) Schematic representation of the thermal microdevice. (b) Gas adsorbs into the tip filled with zeolites and consequently increases the thermal capacity $\left(C_{t h}\right)$ of the tip. 
A single resistor is used for both heating and temperature measurement thanks to high frequency modulation and capacitive coupling (Capacitively coupled electrical substitution) ([10]). When gas adsorbs in the zeolite layer, the thermal capacity of the tip $C_{t h}$ increases. The thermal capacity is extracted using a closed loop identification algorithm.

\subsubsection{Device fabrication}

The structure is $9 \mu m$ thick made out of a SOI wafer through front-side and back-side etching. The fabrication process is sketched in Fig. 3.
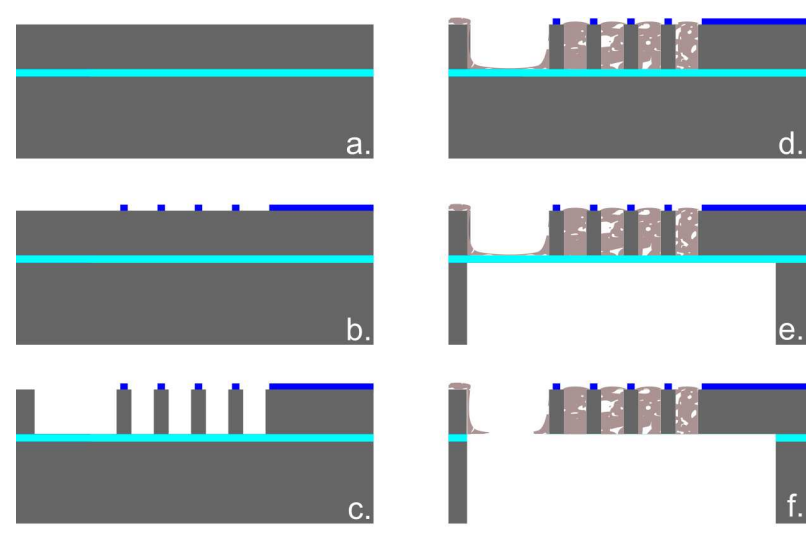

Fig. 3 Microfabrication process.

Starting from a SOI wafer (Fig 3.a), first aluminum is deposited by sputtering, patterned by UV-lithography and dry-etched with chlorine-based plasma to form the aluminum track on the front side (Fig 3.b). Next, $\mathrm{SiO}_{2}$ and aluminum are subsequently sputtered as hard mask materials and the MEMS device shape is patterned by lithography and subsequent RIEs of $\mathrm{Al}$ and $\mathrm{SiO}_{2}$. The wafer is etched by Si deep RIE (Bosch's process) down to the buried oxide layer. Then, mask $\mathrm{Al}$ and $\mathrm{SiO}_{2}$ layers are removed. At this step, the front side topology is defined (Fig 3.c). A zeolite embedding into deep trenches technique was specifically developed for this project (Fig 3.d). The technique combines successive dispensing and calcination steps repeated three to four times for a complete filling. Liquid droplets with zeolite suspension are dispensed on a cantilever, and the chip is readily heated on a hotplate at $320^{\circ} \mathrm{C}$ for drying. To release the cantilever, the handling silicon substrate is etched out by deep RIE from the back side (Fig 3.e), instead of $\mathrm{SiO}_{2}$ etching with hydrofluoric (HF) acid, which is a more standard method for releasing the cantilever. The purpose is to prevent any zeolite damaging. The etching $\mathrm{SiO}_{2}$ of the buried oxide layer with $\mathrm{CHF}_{3}$ RIE is performed as much as possible (Fig 3.f).
FAU-type zeolite with a particle size of $10-20 \mathrm{~nm}$ was prepared according to the procedure described in detail elsewhere ([11]). Hydrophilic FAU-type zeolites were used for water molecule detection. The zeolite $\mathrm{X}$ with FAU type framework structure (FAUX) was synthesized from a clear precursor suspension with a molar composition: $9 \mathrm{Na}_{2} \mathrm{O}$ : $1.1 \mathrm{Al}_{2} \mathrm{O}_{3}$ : $10 \mathrm{SiO}_{2}$ : $45 \mathrm{H}_{2} \mathrm{O}$. The clear precursor suspension was stabilized via control mixing of two solutions, i.e., silica- and aluminabased dissolved in $\mathrm{NaOH}$ and $\mathrm{H}_{2} \mathrm{O}$. The final precursor suspension was aged for $24 h$ at room temperature, and then subjected to hydrothermal crystallization at $50^{\circ} \mathrm{C}$ for $45 h$. The solid products (nanosized FAU-X zeolite) were recovered by centrifugation (25000 rpm for $4 h$ ), purified with $\mathrm{H}_{2} \mathrm{O}$ until $\mathrm{pH}=7$ and then freezedried. The FAU-X zeolite nanocrystals can absorb up to $32 \%$ water, as revealed by thermogravimetry. The high hydrophilicity of the FAU-X crystals is due to the high aluminum content $(\mathrm{Si} / \mathrm{Al}=1)$.

\subsubsection{Device characterization}

The single track enables lowering the thermal conductance of the cantilever to the bulk with less metal and narrower supporting beam. The aluminum-conducting track had a resistance of $25.6 \Omega$ and a TCR of $3.610^{-3} / \mathrm{K}$. The silicon supporting structure (sensor device) has a dimension of $9 \mu \mathrm{m}$ thick and $200 \mu \mathrm{m}$ diameter. The hollow silicon supporting structure enhances the zeolite to silicon mass ratio, thus improving the relative thermal capacity variation under gas adsorption. The thermal capacity of the silicon structure was $0.5 \mu \mathrm{J} / \mathrm{K}$ without zeolites, and $3.6 \mu \mathrm{J} / K$ with zeolites at ambient conditions. A silicon beam, $250 \mu \mathrm{m}$ long and $15 \mu \mathrm{m}$ wide, links the discus shape tip to the bulk silicon and it acts as the weak thermal link with a thermal conductance of $60 \mu W / K$.

\subsection{Conditioning electronics}

The analogous conditioning electronics equilibrates a resistor bridge including a measurement device and a reference device (see ([10]) for details), and amplifies the signal by a factor 10000 before 12-bit analog-todigital conversion by the micro-controller.

\subsection{Identification algorithm}

The CLSRIVC algorithm aims at identifying a continuoustime model from finite sequences $\left\{r\left(t_{k}\right)\right\}_{k=1}^{N},\left\{P\left(t_{k}\right)\right\}_{k=1}^{N}$ and $\left\{V\left(t_{k}\right)\right\}_{k=1}^{N}$ where $r\left(t_{k}\right), P\left(t_{k}\right)$ and $V\left(t_{k}\right)$ denote 


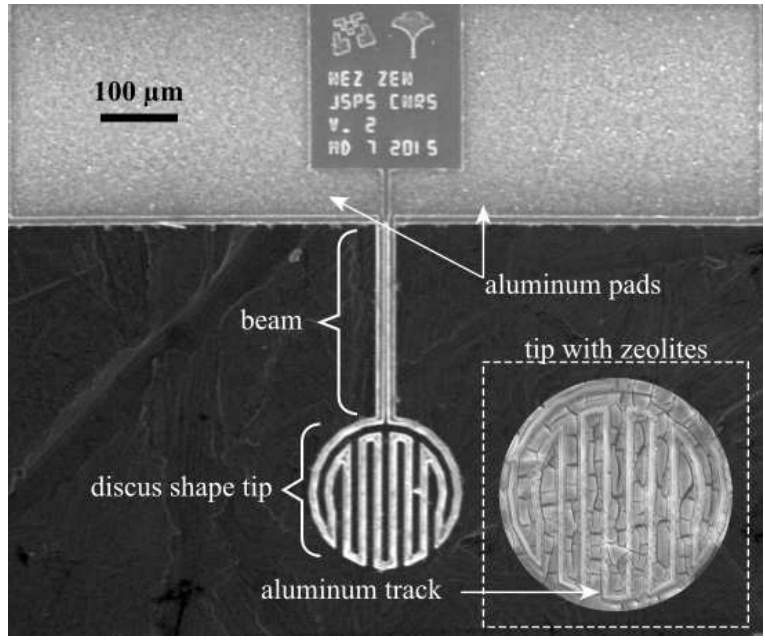

Fig. 4 SEM pictures of a micro-fabricated silicon $(250 \mu \mathrm{m}$ beam and $200 \mu m$ diameter) discus tip structure with a thickness of $9 \mu \mathrm{m}$; the single aluminum track serves as both the heater and the temperature sensor. Bottom left inset: the tip filled with FAU-type zeolite.

the sampled value of the PRBS binary sequence, the Joule power and the voltage representative of the temperature at time instant $t_{k}$ around the operating point. The implementation of the algorithm requires also the knowledge of the controller.

Remark 1 : The thermal device is operated in closedloop using a PI controller. $P\left(t_{k}\right)$ is then computed as follows:

$P\left(t_{k}\right)=x\left(t_{k}\right)+r\left(t_{k}\right)$

with $x\left(t_{k}\right)=x\left(t_{k-1}\right)+k_{p}\left(-V\left(t_{k}\right)\right)+\left(k_{p}-k_{i}\right)\left(-V\left(t_{k-1}\right)\right)$ where $k_{p}$ and $k_{i}$ are the parameters of the controller.

Let us denote $\theta$ the parameter vector of the continuoustime model (1):

$\theta=\left(\begin{array}{c}\tau_{t h} \\ G_{0} G_{e}\end{array}\right)$

The CLSRIVC algorithm is an instrumental variable based algorithm. From the fact that the instrument is computed from the noise free simulation of the closed loop, the implementation of the CLSRIVC algorithm requires the use of an iterative scheme.

The estimate for $\theta$ at the iteration $i$ is denoted $\hat{\theta}_{i}$ and is given by:

$\hat{\theta}_{i}=\left(\sum_{k=1}^{N} \widehat{\varphi}_{f / t_{k}} \varphi_{f / t_{k}}^{T}\right)^{-1} \sum_{k=1}^{N} \widehat{\varphi}_{f / t_{k}} V_{f / t_{k}}$

where $V_{f / t_{k}}, \varphi_{f / t_{k}}$ and $\widehat{\varphi}_{f / t_{k}}$ are built as follows
$-V_{f / t}=f_{c}(p) V_{t}$

with $f_{c}(p)$ a low-pass stable filter;

$-\varphi_{f / t}=f_{c}(p) \varphi_{t}$

with $\varphi_{t}=\left(\begin{array}{c}-\dot{V}_{t} \\ P_{t}\end{array}\right)$

$-\widehat{\varphi}_{f / t}=f_{c}(p) \widehat{\varphi}_{t}$

with $\widehat{\varphi}_{t}=\left(\begin{array}{c}-\hat{\dot{V}}_{t / \hat{\theta}_{i-1}} \\ \widehat{P}_{t / \hat{\theta}_{i-1}}\end{array}\right)$.

$\widehat{\dot{V}}_{t / \hat{\theta}_{i-1}}$ (respectively $\widehat{P}_{t / \hat{\theta}_{i-1}}$ ) corresponds to the estimation of $\dot{V}_{t}$ (respectively $P_{t}$ ) via a simulation of the closed loop using the estimated parameter vector $\hat{\theta}_{i-1}$ obtained at the previous iteration.

About the choice of the low-pass stable filter $f_{c}(p)$, it has been shown in [8] that, for the CLSRIVC algorithm, the optimal filter is $f_{c}(p)=\frac{1}{1+\tau_{t h} p}$. The following iterative scheme is then proposed:

1. Choose $f_{c}(p)=1$ and apply the CLSRIVC algorithm with (5).

This provides $\hat{\theta}_{0}$ and then a first estimation of the thermal capacity $\tau_{t h}$ : the first component of $\hat{\theta}_{0}$ i.e. $\hat{\theta}_{0}(1)$;

2. Given this first estimation, design the filter $f_{c}(p)=$ $\frac{1}{1+\hat{\theta}_{0}(1) p}$;

3. Apply the CLSRIVC algorithm to get a new estimate $\hat{\theta}_{1}$;

4. Repeat steps 2 and 3 until convergence of step 3 or until a finite number of iterations.

Compute the variance of the estimated parameters from the Cramer-Rao bound with the last estimated parameter vector.

The first step is an initialization step: an initial estimate of the model is necessary so as to implement the CLSRIVC algorithm. Then at each iteration a intermediate model is used to design the low-pass filter. The convergence of the algorithm has not yet been proved theoretically, however it is quite similar to some iterative least squares algorithms for which the default number of iterations in MATLAB is 20 .

The implementation of the algorithm requires the choice of several parameters. The first one is the number of samples $N$. The choice of $N$ is the result of a compromise. If $N$ is high, then the variance of the estimation is reduced, however a local variation of the thermal capacity may not be detected. If $N$ is low, then a local variation of the thermal capacity can be detected, however if this variation is low, then it may not be detected due to the higher variance. 


\subsection{Experimental Set-up}

Two thermal devices are placed in a gas sensor cell with a size of $7 \mathrm{~cm}^{3}$ (Fig. 5). One device, without zeolite coating, is used as a reference for differential measurement. Such configuration allow differential measurement in order to reject effects of ambient temperature variation or second order physical quantities resulting in common mode perturbations (e.g. pressure, vibration).

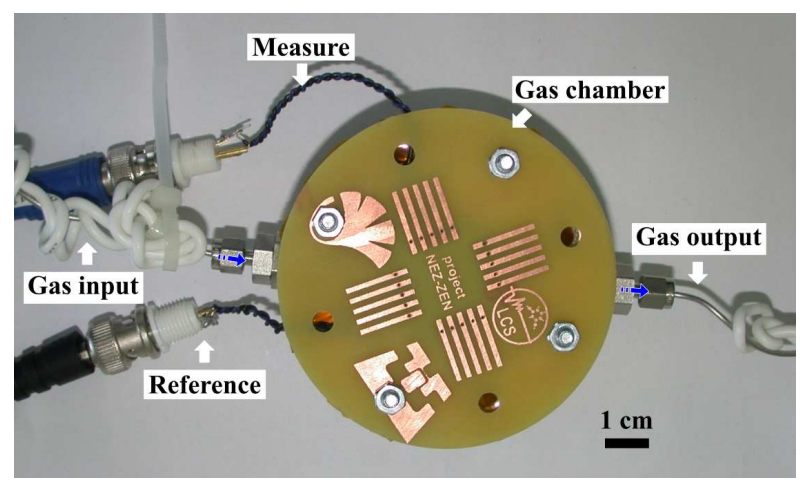

Fig. 5 Picture of the sensor cell. Two devices are placed in an hermetically sealed cell. Two gas connections enables gas flow in the cell. The sensor cell is an $8 \mathrm{~cm}$ diameter aluminum cylinder with a $7 \mathrm{~cm}^{3}$ cavity chamber buried. The cavity chamber is covered by an epoxy board on which the thermal devices are sealed and wire-bonded. Polymer joint and screws ensure hermetical sealing of the chamber. Line inlet and outlet for flow passing are heated to prevent condensation.

The sensor cell was connected to the gas flow set-up, which guarantees controlled concentrations of analytes (Fig. 6). The analyte, here water, is delivered in an argon flow ([12]). The concentration of analytes is controlled through the temperature of the thermostated bath and the velocity of the flow passing therein in order to obtain the appropriate parameters of the Antoine equation $([13])$. The analyte is chosen in accordance to the selectivity of the sensor device, i.e., the hydrophilic FAU-type zeolite is expected to be highly sensitive to water. Recordings have been carried out at atmospheric pressure under a constant flow $\left(20 \mathrm{ccmin}^{-1}\right)$, and the inox gas tubings were heated to prevent analyte from condensation during the measurements.

\subsection{Operation mode}

In this work, closed-loop operation is chosen to keep the temperature of the thermal micro-device constant. Open and closed-loop modes are illustrated in Fig. 7 with the recorded signals under self-stimulation by a

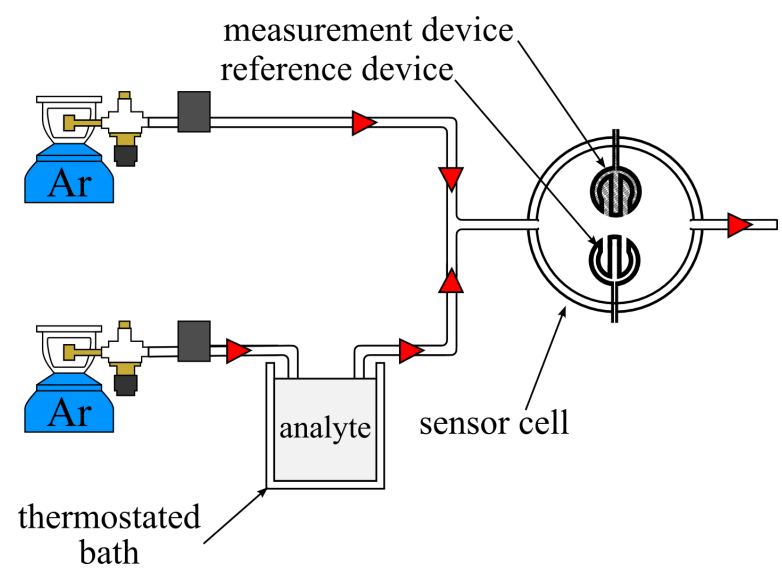

Fig. 6 Sketch of the experimental set-up.

pseudo-random binary sequence (PRBS) of heat pulses. In open-loop the temperature varies upon the heat stimulation highlighting the thermal constant. Gas detection through thermal capacity extraction in open-loop mode was discussed in ([4]).

In closed-loop mode, the temperature is kept constant. When pseudo-random stimulation is produced, the feedback controller compensates for induced temperature variations. The device thermal parameters $\left(G_{t h}\right.$, $\left.C_{t h}\right)$ are extracted using the identification algorithm, from the PRBS stimulation, the temperature signal and the power signal.

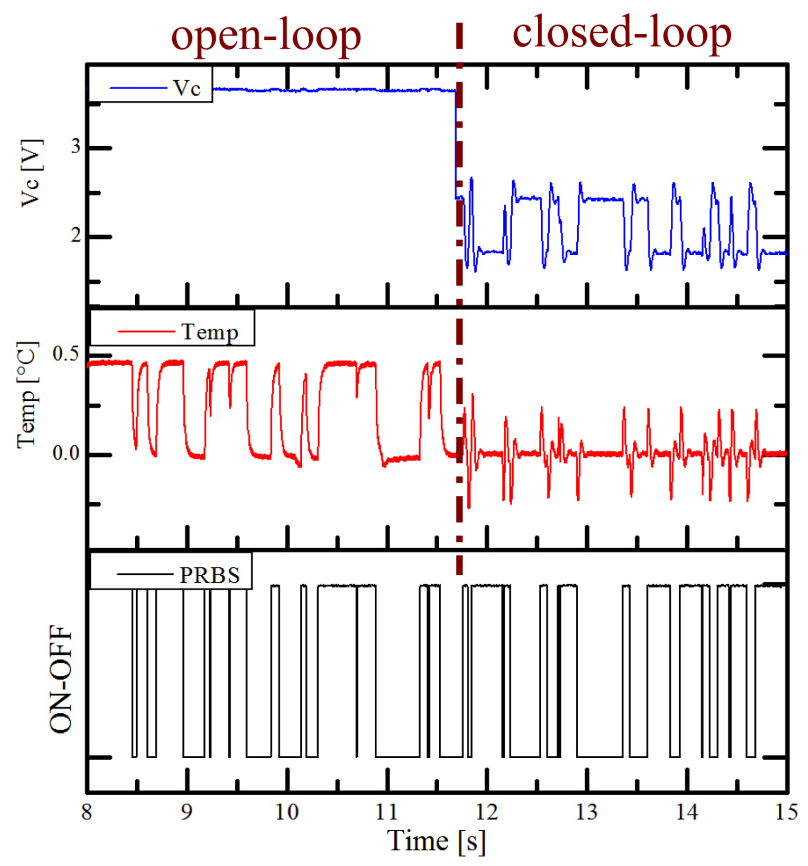

Fig. 7 Illustration of open-loop and closed-loop mode operation of the thermal device. 
Experiments with various analyte concentrations were undergone to demonstrate the parameter extraction. Water was used as analyte because of specific adsorbance by hydrophilic FAU-type zeolite. Recordings were carried out at atmospheric pressure and room temperature under a constant flow. The measure is differential between measurement device and reference device without zeolites to reject secondary effects such as room temperature variations.

Remark 2 : About the operation mode, it would be interesting to compare performances of open-loop and closed-loop identification, however it is currently difficult on the considered identification problem, for two main reasons. Firstly, a hard constraint in our operation mode is to keep constant the temperature of the device. Secondly, the input/output signals must remain around the operating point of interest in order to give meaning to the linear approximation (1). These two constraints imply the identification in the closed-loop mode.

\section{Results, parameter extraction with identification algorithm}

Typical results obtained in closed-loop mode operation with self-stimulation and parameter extractions are illustrated in this section. The CLSRIVC algorithm has been implemented on sequences of $120 \mathrm{~s}$, the sampling period has been chosen equal to $5 \mathrm{~ms}$.

Fig. 8 presents the real temperature variation around the operating point (blue curve) and its estimation with the estimated continuous-time model (red curve). It appears that the model obtained with the CLSRIVC algorithm describes correctly the dynamical behavior of the closed-loop.

Fig. 9 and 10 present the extracted thermal capacity when concentration varies from 0 to $10000 \mathrm{ppm}$ (blue curve). Amplitude of the thermal capacity increases with concentration as expected from the proposed detection principle. The response time $(\sim 10 \mathrm{~min})$ corresponds to the filling of the tubing, the sensor cell and the adsorption of water molecules in the zeolite layer. Long term stability and level reproducibility should be noticed.

The standard deviation (std) on the estimated thermal capacity has also been estimated from the CramerRao bound. This allows the characterization of an un-

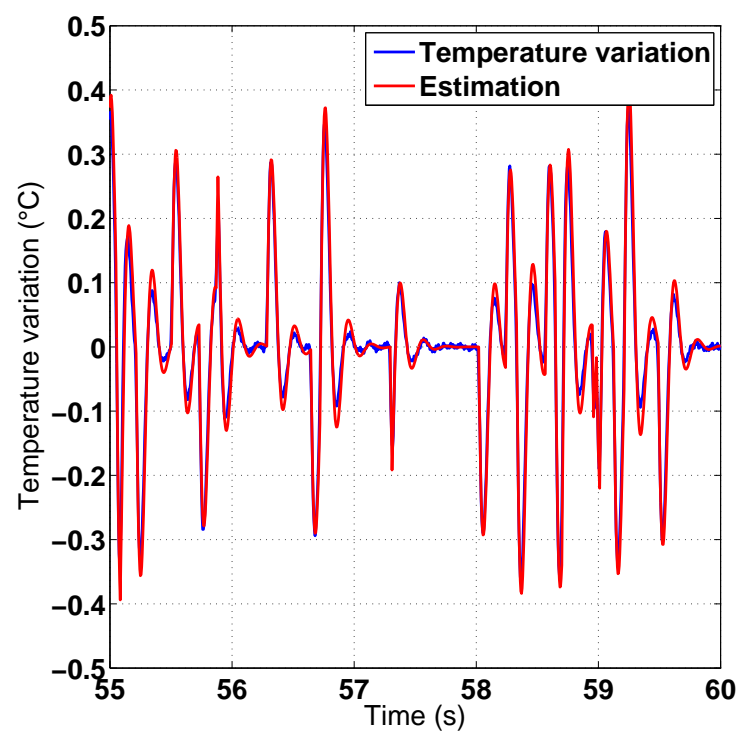

Fig. 8 Comparison between the temperature variation around the operating point and its estimation.

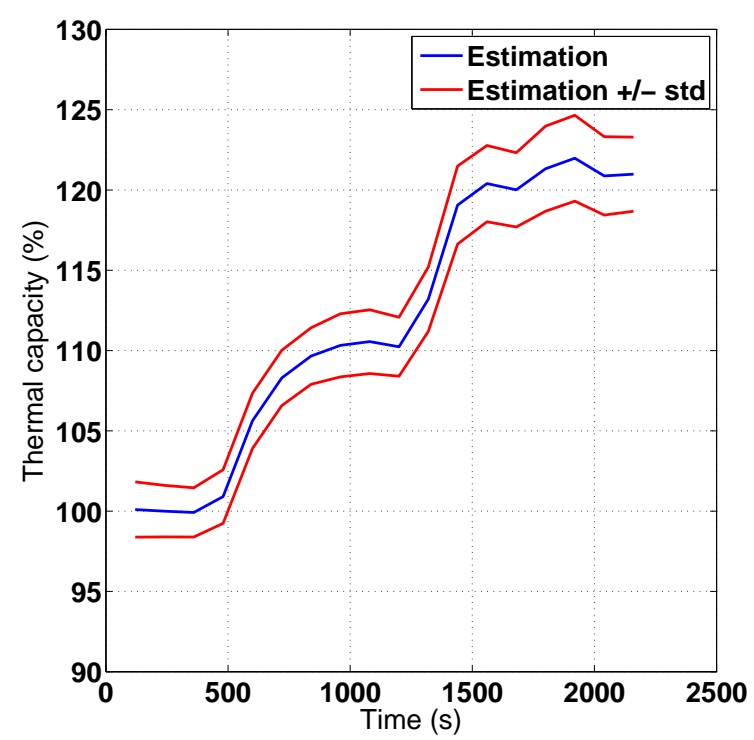

Fig. 9 Evolution of the extracted thermal capacity parameter through self-identification procedure in closed-loop mode upon the concentration of water vapor in argon flow; successively 0ppm, 5000ppm, 10000ppm.

certainty interval on the estimated thermal capacity as depicted on Fig. 9 and 10 (red curve).

Closed-loop mode operation is attractive since it enables to control the dynamic performance of the device, speed and measurement range, as well as the operating point. Therefore, the ability to extract the thermal pa- 


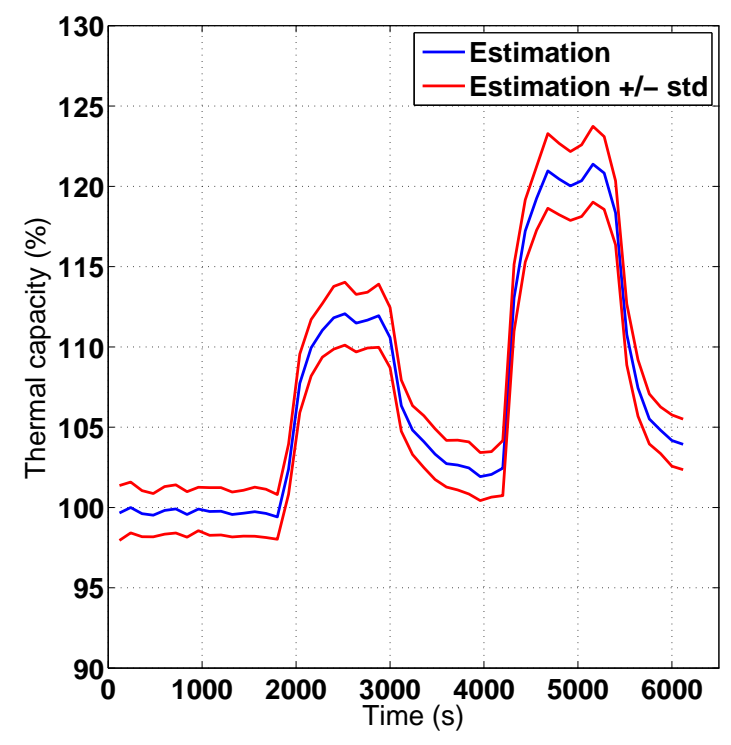

Fig. 10 Evolution of the extracted thermal capacity parameter through self-identification procedure in closed-loop mode upon the concentration of water vapor in argon flow; successively 0ppm, 5000ppm, 0ppm, 10000ppm, 0ppm.

rameter variations due to gas adsorption while operating the device in closed-loop mode is of great interest to allow taking advantage of the operation mode. Furthermore, constant temperature operation of the device can be favorable as it reduces convection around the tip of the device.

Remark 3 : A drift on the thermal capacity estimation can be observed on Fig. 10. This is due to the fact that desorption time is slower than adsorption time and to the fact that, in the experiment illustrated in Fig. 10, the steady state for 0ppm has not been reached after $1000 \mathrm{~s}$, when the concentration step occurred. Future work involving smaller, and hence faster thermal devices, will enable experiments with more cycles and getting back to steady step within a shorter time.

\section{Conclusion}

Thermal capacity variation du to gas adsorption onto zeolite selective coating was performed with and identification algorithm while operating in closed-loop mode. The implemented identification algorithm is a specific closed-loop identification algorithm allowing an unbiased estimation of the thermal capacity. Extracted thermal capacity varies as expected with the concentration of a gas.
Future work will involve further miniaturization of the device in order to reduce the time constant du to gas adsorption and to improve the sensitivity with lower thermal conductance.

Acknowledgements The project was supported by the French and Japanese bilateral exchange program PRC JSPS-CNRS and Normandy C2-MTM Project. Micro-fabrication process was done in the Takeda clean room at the University of Tokyo, partially financed by the MEXT Nanotechnology Platform.

\section{References}

1. F.M. Battiston, J.-P. Ramseyer, H.P. Lang, M.K. Baller, Ch. Gerber, J.K. Gimzewski, E. Meyer, H.-J. Guntherodt, A chemical sensor based on a microfabricated cantilever array with simultaneous resonance frequency and bending readout, Sensors and Actuators B vol. 77, pp. 122-131 (2001)

2. R. Berger, Ch. Gerber, J. K. Gimzewski, Thermal analysis using a micromechanical calorimeter, Appl. Phys. Lett, vol. 69(1), pp. 40-42 (1996)

3. L.R. Senesac, D. Yi, A. Greve, J.H. Hales, Z.J. Davis, D.M. Nicholson, A. Boisen, T. Thundat, Microdifferential thermal analysis detection of adsorbed explosive molecules using microfabricated bridges, Rev. Sci. Instr, vol. 80, 035102 (2006)

4. M. Denoual, M. Pouliquen, D. Robbes, O. de Sagazan, J. Grand, H. Awala, S. Mintova, S. Inoue, A. Mita-Tixier, Y. Mita, Microfabricated test structures for thermal gas sensors, Proc. of ICMTS (2016)

5. M. Denoual, D. Robbes, S. Inoue, Y. Mita, J. Grand, H. Awala, S. Mintova, Thermal Resonant Zeolite-based Gas Sensor, Sensor and Actuators B: Chemical, vol. 245, pp. 179-182 (2017)

6. P. Van den Hof. Closed-loop issues in system identification. Annual Reviews in Control, 22, pp. 173-186 (1998)

7. L. Ljung, System identification: theory for the user, Prentice Hall (1999)

8. M. Gilson, H. Garnier, P. Young, P. Van den Hof, Instrumental variable methods for closed-loop continuous-time system identification, in Identification of Continuous-Time Models from Sampled Data, H. Garnier, L. Wangs SpringerVerlag, London, pp. 133-160 (2008)

9. M.A. Urbiztondo, A. Peralta, I. Pellejero, J. Ses, M.P. Pina, I. Dufour, J. Santamaria, Detection of organic vapours with $\mathrm{Si}$ cantilevers coated with inorganic (zeolites) or organic (polymer) layers, Sensors and Actuators B, vol. 171-172, pp. 822-831 (2012)

10. M. Denoual, G. Allègre, S. Delaunay, D. Robbes, Capacitively coupled electrical substitution for resistive bolometer enhancement, Meas. Sci. Technol., vol 20, 015105 (2009)

11. H. Awala, J-P. Gilson, R. Retoux, P. Boullay, J-M. Goupil, V. Valtchev, S. Mintova, Template-free nanosized faujasite-type zeolites, Nature Mater., vol 14, pp. 447-451 (2015)

12. S. Thomas, P. Bazin, L. Lakiss, V. de Waele, S. Mintova, In Situ Infrared Molecular Detection Using PalladiumContaining Zeolite Films, Langmuir, vol 27, pp. 1468914695 (2011)

13. G. W. Thomson, The Antoine Equation for Vaporpressure Data, Chem. Rev., vol 38, pp. 1-39 (1946) 